\title{
Author Correction: Association analysis in over 329,000 individuals identifies 116 independent variants influencing neuroticism
}

Michelle Luciano (D), Saskia P. Hagenaars, Gail Davies, W. David Hill, Toni-Kim Clarke, Masoud Shirali (D), Sarah E. Harris, Riccardo E. Marioni, David C. Liewald (D), Chloe Fawns-Ritchie, Mark J. Adams, David M. Howard (D), Cathryn M. Lewis (D), Catharine R. Gale, Andrew M. McIntosh (D) and lan J. Deary

Correction to: Nature Genetics https://doi.org/10.1038/s41588-017-0013-8, published online 18 December 2017.

In the version of this article initially published, in Table 2, the descriptions of pathways and definitions in the first and last columns did not correctly correspond to the values in the other columns. The error has been corrected in the HTML and PDF versions of the article.

Published online: 29 January 2019

https://doi.org/10.1038/s41588-019-0357-3

\section{Publisher Correction: An evolutionary framework for measuring epigenomic information and estimating cell-type-specific fitness consequences}

\section{Brad Gulko $[$ and Adam Siepel(1)}

Correction to: Nature Genetics https://doi.org/10.1038/s41588-018-0300-z, published online 17 December 2018.

In the version of this article initially published, in the Methods section 'Statistics and data analysis', subsection 'Measuring entropy with INSIGHT, the equation for the maximized log likelihood incorrectly duplicated the equation for entropy; the equation read $H(X)=-C \sum_{x} P(x \mid \hat{\theta}) \log P(x \mid \hat{\theta})$ but should have $\operatorname{read} \mathcal{L}(\hat{\theta}, X)=\max _{\theta} \log P(X \mid \theta)=\max _{\theta} \sum_{i} \log P\left(X_{i} \mid \theta\right)$. The error has been corrected in the HTML and PDF versions of the article. 